\title{
Intraglomerular metastasis: a necropsy study
}

\author{
Desu Sridevi, Dhanpat Jain, Rakesh K Vasishta, Kusum Joshi
}

\begin{abstract}
Intraglomerular metastasis is a rare phenomenon. Four cases are reported here. The primary malignancies in these four cases were squamous cell carcinoma of the lung, adenocarcinoma of pancreas, haematological malignancy with a malignant intrapulmonary teratoma, and undifferentiated pleural mesothelioma. There was no significant renal functional impairment in any case except for mild proteinuria in one. Histopathology showed tumour cells in the mesangium, in the glomerular tufts replacing the endothelial cells, as well as in an extracapillary location replacing the parietal cells. In one case, tumour cells formed crescent-like structures. Such tumour deposits were better appreciated with special stains. The presence of intraglomerular metastasis indicates dissemination of the malignancy and hence a poor prognosis even though there is no significant impairment of renal function.

(f Clin Pathol 1999;52:307-309)
\end{abstract}

Keywords: kidney; tumour metastasis; glomerulus

The presence of metastatic tumour cells in segments of renal glomeruli-that is, intraglomerular metastasis-is a rare phenomenon, with only a few published case reports. ${ }^{1-4}$ Most of the cases have been detected at necropsy. In patients presenting with proteinuria, intraglomerular metastasis detected on kidney biopsy has occasionally been the first indication of the presence of malignancy. In this study we investigated the occurrence of intraglomerular metastasis in cases of disseminated malignancies, and to correlate it with the clinical features, especially renal function.

\section{Methods}

We examined necropsies performed in the department of pathology, Post Graduate Institute of Medical Education and Research, Chandigarh, between January 1983 and December 1993. We examined a minimum of two blocks (approximately $1.5 \mathrm{~cm}^{2}$ ) from each kidney in all cases showing disseminated malignancy. Five micron thick sections stained with haematoxylin and eosin (H\&E) were studied. The total number of glomeruli in each section was counted and the percentage of glomeruli showing tumour cells was calculated. In cases showing intraglomerular metastasis, appropriate special stains were done-for example, periodic acid Schiff (PAS), Alcian blue, Masson's trichrome, and chloroacetate esterase. In one case, indirect immunoperoxidase staining for carcinoembryonic antigen (CEA) was also performed. Clinical records of these patients were analysed, specifically with regard to renal function.

\section{Results}

During the study period, of total of 5050 necropsies performed, 136 cases showed evidence of a disseminated malignancy. Macroscopic or microscopic metastatic deposits in the kidneys were seen in 42 of these, but only four cases showed intraglomerular metastasis. The details of the four cases are summarised in table 1 .

In the solid malignancies (cases 1, 2, and 4), tumour cells were seen in the glomerular tufts, replacing the endothelial cells, and also in the mesangial matrix. These appeared as nodular lesions (figs 1 and 2). Case 2, in addition, showed extracapillary extension of the tumour cells into the Bowman's space, occasionally forming "crescent"-like clusters of tumour cells (fig 2A). Tumour cells were also seen replacing the parietal epithelium in places. In case 1, four sections examined showed a nodular deposit in only one glomerulus of 400 glomeruli counted. PAS stain showed that the deposit was adjacent to the mesangium on one aspect and partly surrounded by basement membrane on the other. In the haematological malignancy (case 3), solitary tumour cells were seen in the glomerular tufts and in the capillary lumina, as well as near the parietal epithelium (fig 3). This was an unusual case of cellular phase of myelofibrosis with myeloid metapla6 January 1999

Table 1 Details of the cases showing intraglomerular metastasis

\begin{tabular}{|c|c|c|c|c|c|}
\hline \multirow[b]{2}{*}{ Case No } & \multirow[b]{2}{*}{ Primary malignancy } & \multirow[b]{2}{*}{ Other sites of metastases } & \multicolumn{2}{|c|}{ Glomeruli involved } & \multirow{2}{*}{$\begin{array}{l}\text { Presence of parenchymal deposits in } \\
\text { kidneys }\end{array}$} \\
\hline & & & On $H \mathcal{E} E$ & After special stains & \\
\hline 1. $47 / \mathrm{M}$ & Squamous cell carcinoma (lung) & Brain, adrenals & Single & Single & Multiple, 0.5 to $2.5 \mathrm{~cm}$ diameter \\
\hline 2. $42 / \mathrm{M}$ & Pancreatic adenocarcinoma & $\begin{array}{l}\text { Liver, lungs, heart, adrenal, } \\
\text { gallbladder, lymph node }\end{array}$ & $22 \%$ & $40 \%$ & Multiple, 0.5 to $1.0 \mathrm{~cm}$ diameter \\
\hline 3. $21 / \mathrm{M}$ & $\begin{array}{l}\text { Haematological malignancy (malignant } \\
\text { intrapulmonary teratoma associated with cellular } \\
\text { phase of myelofibrosis with myeloid metaplasia }\end{array}$ & $\begin{array}{l}\text { Liver, spleen, lung, hilar } \\
\text { lymph nodes, adrenal }\end{array}$ & $18 \%$ & $28 \%$ & Microscopic infiltration only \\
\hline 4. $14 / \mathrm{M}$ & Undifferentiated mesothelioma (lung) & $\begin{array}{l}\text { Regional lymph nodes, lung, } \\
\text { adrenals, liver }\end{array}$ & $10 \%$ & $12 \%$ & None \\
\hline
\end{tabular}




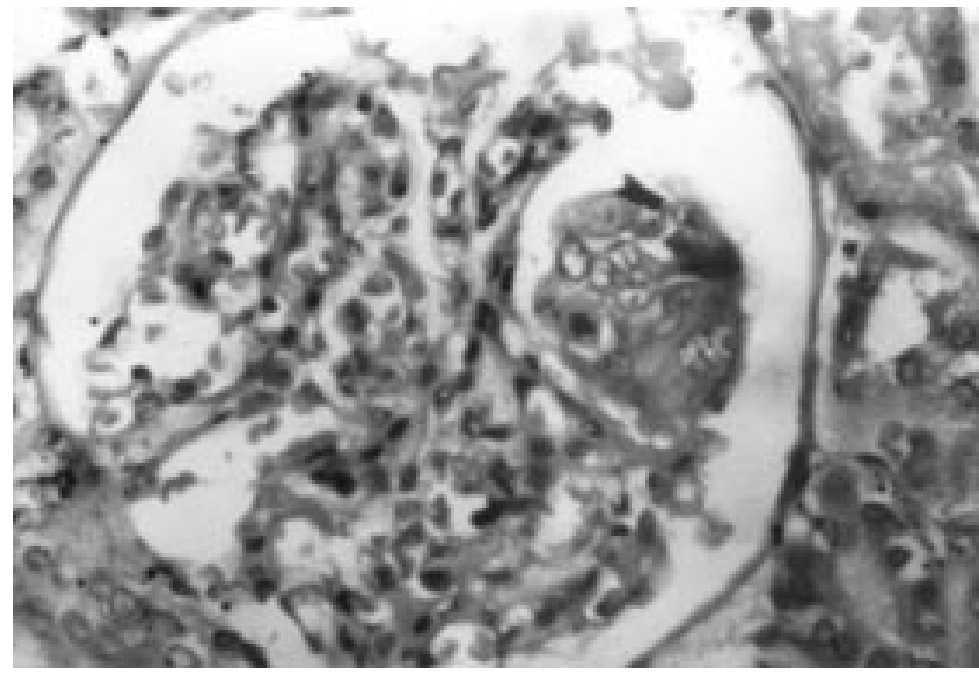

Figure 1 Nodular tumor deposits in the glomerulus in case 1 (haematoxylin and eosin, 636).

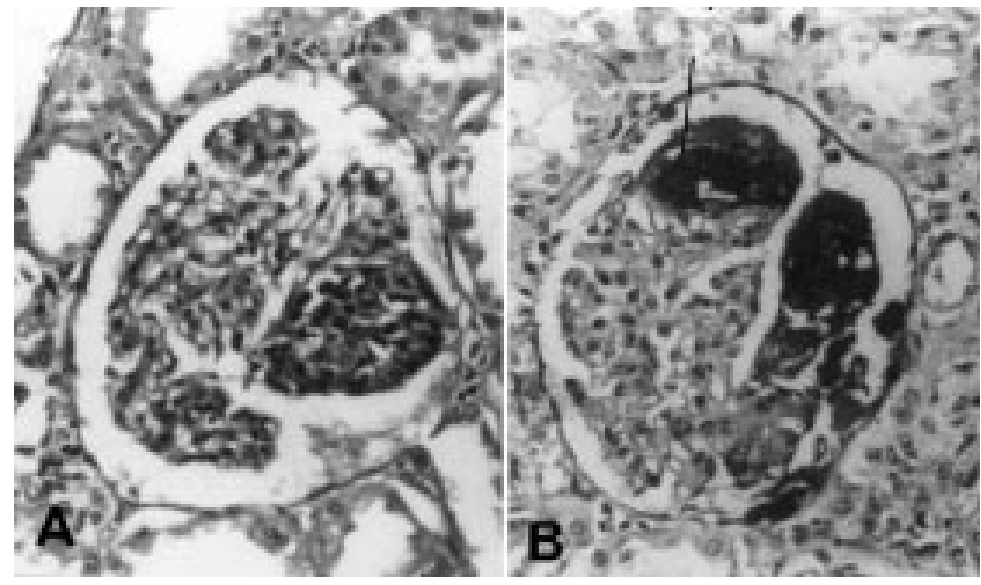

Figure 2 (A) Nodular tumor deposits in glomerular tufts and crescent-like collections in Bowman's space in case 2 (haematoxylin and eosin, 550); (B) strong positivity in the tumor deposits for CEA (indirect immunoperoxidase stain, 550).

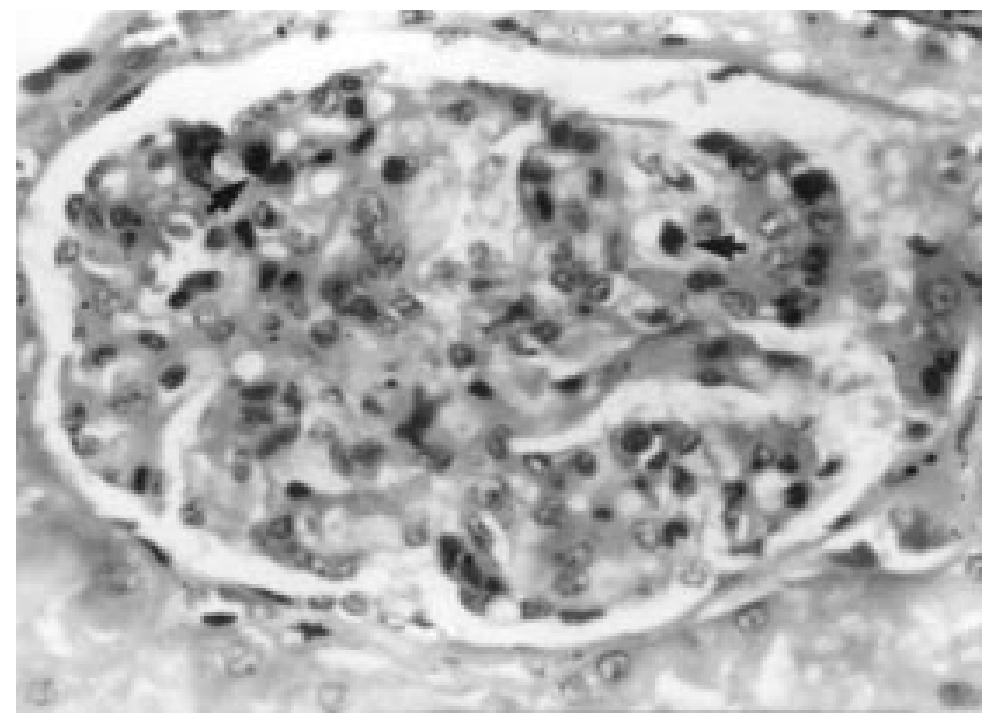

Figure 3 Discrete tumour cells (arrows) in the glomerulus in case 3 (haematoxylin and eosin, 629). many more deposits were identified on special stains, as compared to $\mathrm{H} \& \mathrm{E}$ stained slides. In case 4 , though the tumour cells were negative for all special stains, the tumour deposits were better identified on PAS stain, as the intracapillary tumour deposits were delineated by the basement membrane. Staining for chloroacetate esterase in case 3 clearly outlined positive tumour cells.

Biochemical and microscopic routine urine examination, blood urea, serum creatinine, and blood pressure were within normal limits in the all cases except for traces of albumin in the urine in one case (case 2).

\section{Discussion}

The affinity of certain malignant tumors for metastasis to specific organs or tissues is well known, though unexplained. While circulating tumour cells are common in any malignancy, these rarely seed the distant tissues to give rise to metastatic deposits. In spite of receiving a large part of the blood supply, the renal glomeruli are very uncommon sites of metastasis in a malignancy. The unusual occurrence of intraglomerular metastasis raises questions such as the relative incidence of this type of renal metastasis, the morphological patterns of involvement, the mechanism of spread, the symptoms and signs of glomerular involvement, and the its clinical implications.

The exact incidence of this type of metastasis is difficult to ascertain as there are only a few case reports. In the largest published series by Wagle et $a l,{ }^{2}$ intraglomerular metastasis constituted $6 \%$ of all secondary carcinomas of the kidneys. In the present study, it was found in $10 \%(4 / 42)$ of all secondary neoplasms of the kidney, and 3\% (4/136) of all cases of disseminated malignancies. Of the published cases, the common primary sites reported have been the skin and lung. ${ }^{136}$ In this study, two cases had a primary in the lung.

In 1960, Wuketich ${ }^{1}$ described two patterns of intraglomerular metastasis: a diffuse "intracapillary" variety in which tumour cells grow diffusely in all the glomerular tufts, and a diffuse "extracapillary" type in which the parietal epithelium of the Bowman's space is replaced by the tumour cells. We observed intracapillary pattern of metastasis in cases 1,2 , and 4 , appearing as nodular lesions. Case 2 also showed extracapillary pattern in the form of discrete cells, and crescents of tumour cells in the Bowman's space (fig 2A). Appropriate special stains helped to identify the tumour cells better, and a larger number of glomeruli appeared to be involved than apparent on the routine $H \& E$ stain. Even when endothelial cells have been replaced by tumour cells, this does not seem to interfere with the glomerular basement membrane function since no significant renal function impairment was seen. Ultrastructural examination was not carried out in the present study, but Belghiti et al have reported a normal basement membrane in a case of intraglomerular metastasis. ${ }^{4}$

While the exact mechanism of spread in this fashion is not known, many mechanisms have been hypothesised. ${ }^{67}$ Allen $^{6}$ proposed two posnary teratoma.

In case 2 , the tumour cells were positive for Alcian blue staining and CEA (fig 2B) and 
sible mechanisms: intralymphatic invasion, and intravascular spread from arterial/venous tumour emboli. The first claim is refuted as lymphatics have not been demonstrated in glomeruli, ${ }^{8}$ but the second mechanism seems more probable. The narrow calibre and the slower blood flow in the glomerular capillaries probably enable the tumour cells to attach to capillary wall, and form metastatic deposits.

In cases of tumour infiltration in the kidneys, the organ size is usually preserved and $30 \%$ of the patients have microscopic haematuria. ${ }^{2}$ In cases of intraglomerular metastasis, invariably there is proteinuria with or without haematuria or significant impairment of renal function. ${ }^{29}$ Unlike paraneoplastic nephrotic syndrome, proteinuria from intraglomerular metastasis spells a poor prognosis for the patient because it implies dissemination of the primary malignancy. In our study, in the case showing albuminuria the glomeruli were involved in a combined intraand extracapillary pattern of metastasis. The absence of proteinuria or significant impairment of renal function in any of the other cases was probably because of very focal involvement of the glomeruli or a predominant intracapillary localisation of the tumour cells with an intact basement membrane.

The differential diagnosis of intraglomerular metastasis includes intraglomerular adenomatosis, which is a complication of a malignant neoplasm consisting of columnar cell change in the parietal layer of Bowman's capsule. The lesions are not secondary deposits and appear to be a variety of metaplasia. ${ }^{10}$

1 Wuketich ST. Diffuse intraglomerulare Metastasierung bei malignem Melanoblastom. Oncologia (Basel) 1960;13:35562 .

2 Wagle DG, Moore RH, Murphy GP. Secondary carcinomas of the kidney. F Urol 1975;114:30-2.

3 Datta BN. Intraglomerular metastasis. Indian $\mathcal{f}$ Pathol Microbiol 1978;21:184-5.

4 Belghiti D, Hirbec G, Bernandin JF, et al. Intraglomerular metastases. Cancer 1984;54:2309-12.

5 Kakkar N, Vasishta RK, Banerjee AK, et al. Primary pulmonary malignant teratoma with yolk sac element associated nary malignant teratoma with yolk sac element associa

6 Allen AC. The kidneys: medical and surgical diseases. London: Churchill, 1951:555-65.

7 Willis RA. Secondary tumors of the kidneys. In: The spread of tumours in the human body, 3rd ed. London: Butterworths, 1973:193.

8 Peirce EC. Renal lymphatics. Anat Rec 1944;90:315-35. 9 Fer MF, McKinney TD, Richardson RL, et al. Cancer and 1981;71:704-18.

10 Gordon A. Renal glomerular adenomatosis. f Pathol Bacteriol 1962;83:555. 